\title{
Inhaled biguanides and mTOR inhibition for influenza and coronavirus (Review)
}

\author{
STEVEN LEHRER \\ Fermata Pharma Inc., New York, NY 10023-7909, USA \\ Received December 23, 2019; Accepted March 24, 2020
}

DOI: $10.3892 /$ wasj.2020.42

\begin{abstract}
The mammalian target of rapamycin (mTOR) signaling pathway senses and responds to nutrient availability, energy sufficiency, stress, hormones and mitogens to modulate protein synthesis. Rapamycin is a bacterial product that can inhibit mTOR via the PI3K/AKT/mTOR pathway. mTOR signaling is necessary for the development of influenza and modulates the antibody response to provide cross-protective immunity to lethal infection with influenza virus. In one human study, it was found that the treatment of severe H1N1 influenza-related pneumonia with rapamycin and steroids improved the outcome. However, in other studies, immunosuppression with systemic steroids, and possibly rapamycin as well, was associated with an increased morbidity/mortality and a prolonged viral replication. In order to avoid the systemic side-effects, some investigators have postulated that the inhalation of rapamycin would be desirable. However, the inhalation of rapamycin, with its well-documented lung toxicity, could be contraindicated. Another class of drug, biguanides, can also inhibit mTOR, but have no lung toxicity. Biguanides are widely used small molecule drugs prescribed as oral anti-diabetics that have exhibited considerable promise in oncology. During the 1971 outbreak of influenza, diabetic patients treated with the biguanides, phenformin and buformin, had a lower incidence of infection than diabetics treated with sulfonylureas or insulin. Both buformin and phenformin reduce the mortality of influenza in mice; phenformin is less effective than buformin. The inhalation of buformin or phenformin for influenza may be an effective novel treatment strategy that would limit the risk of systemic side-effects associated with biguanides due to the low inhaled dose. Coronavirus disease 2019 (COVID-19) is an infectious disease caused by SARS-CoV-2, a virus closely related to the SARS virus. The disease is the cause of the 2019-2020 coronavirus outbreak. It is primarily spread between individuals via small droplets emitted from infected individuals when breathing or coughing. PI3K/AKT/mTOR
\end{abstract}

Correspondence to: Dr Steven Lehrer, Fermata Pharma Inc., 30 West 60th Street, New York, NY 10023-7909, USA

E-mail: steven@fermatapharma.com

Key words: influenza, biguanides, mammalian target of rapamycin, buformin, inhaled signaling responses play important roles in MERS-CoV infection and may represent a novel drug target for therapeutic intervention strategies. The present review article discusses the effects of biguanides on influenza and coronavirus.

\section{Contents}

1. Introduction

2. mTOR and influenza

3. Biguanides

4. Buformin, phenformin and human influenza

5. Buformin and influenza in mice

6. Inhaled biguanides for influenza

7. Inhaled buformin or phenformin for coronavirus

8. Conclusions and future perspectives

\section{Introduction}

Influenza develops in approximately $20 \%$ of the world's population each year. In the US, 30,000 to 100,000 deaths occur annually due to influenza. The pandemic of 1918-1919 resulted in 50 million to 100 million deaths.

Vaccination is the primary strategy for the prevention of influenza; however, it is not always adequate. The effectiveness of the seasonal influenza vaccine varies by season. For example, during the period between November 23, 2018 to February 2, 2019, the overall adjusted vaccine effectiveness against all influenza virus infection associated with medically attended acute respiratory illness was $47 \%$. For children aged 6 months to 17 years, the overall vaccine effectiveness was $61 \%$ (1). In addition, a growing body of evidence indicates that the protective immune responses triggered by flu vaccines wane in a matter of weeks (2). Antiviral drugs thus form an important part of an effective approach to influenza and are critical to planning for a pandemic (3).

Five drugs are currently available for the treatment or prophylaxis of influenza infections: The adamantanes (amantadine and rimantadine) and the neuraminidase inhibitors (zanamivir and oseltamivir). In 2019, the FDA approved baloxavir marboxil (trade name, Xofluza), a new class of drug which targets the endonuclease function of the viral PA polymerase subunit and prevents the transcription of viral mRNA (4). Despite the success of baloxavir, certain strains 
of influenza A (H3N2) exhibit a reduced susceptibility (5). Additional antiviral drugs are thus required.

\section{2. mTOR and influenza}

The mammalian target of rapamycin (mTOR) signaling pathway senses and responds to nutrient availability, energy sufficiency, stress, hormones and mitogens to modulate protein synthesis. The mTOR pathway is dysregulated in human diseases, particularly in cancers. Rapamycin (sirolimus) is a bacterial product that can inhibit mTOR via AMPK activation and the inhibition of the PI3K/AKT/mTOR pathway (6).

mTOR signaling is necessary for the development of influenza and modulates the antibody response to provide cross-protective immunity to lethal infection with influenza virus. In animal studies, rapamycin was shown to promote cross-strain protection against lethal infection with influenza virus of various subtypes when administered during immunization with influenza virus subtype H3N2 (7). Mitogenic stimulation accelerates influenza-induced mortality in animals by increasing susceptibility of alveolar type II cells to infection, and pre-treatment with rapamycin reverses this effect (8).

In human studies, the treatment of severe H1N1 influenza-related pneumonia with rapamycin and steroids was shown to improve the outcome $(9,10)$. However, other researchers have demonstrated that immune suppression caused by systemic steroids, and possibly rapamycin as well, is associated with an increased morbidity/mortality and a prolonged viral replication (11).

In order to avoid the systemic side-effects, some investigators have postulated that the inhalation of rapamycin would be desirable. Inhalable rapamycin preparations have been formulated and tested on rats $(12,13)$ but never in humans, and for good reason: A side-effect of oral rapamycin is interstitial pneumonitis (14). The inhalation of rapamycin, with its well-documented lung toxicity, is contraindicated.

\section{Biguanides}

Another class of drug, biguanides, can also inhibit mTOR activation but has no lung toxicity. Biguanides are widely used small molecule drugs prescribed as oral anti-diabetics. They include the following: i) Metformin; ii) phenformin, withdrawn from US market because of its propensity to cause lactic acidosis; iii) buformin (1-butylbiguanide), an oral antidiabetic drug of the biguanide class, chemically related to metformin and phenformin; buformin was marketed by the German pharmaceutical company, Grünenthal, as Silubin; and iv) benfosformin, etoformin, tiformin

Metformin activates the 5' AMP-activated protein kinase (AMPK) pathway through liver kinase B1 (LKB1), eventually causing the inhibition of the mTOR pathway and thus, a reduction in protein synthesis and cellular proliferation. Metformin also appears to indirectly reduce AKT activation, through the AMPK-mediated phosphorylation of insulin receptor substrate 1 (IRS-1), causing the inhibition of the mTOR pathway (15).

Biguanides have no known lung toxicity after decades of use in millions of patients. Biguanides are cell proliferation inhibitors, and their use in oncology holds considerable promise $(16,17)$.

\section{Buformin, phenformin and human influenza}

During the 1971 outbreak of influenza (1968 Hong-Kong H3N2 strain), 110 diabetic patients treated with phenformin or buformin (group A) and 79 diabetic patients treated with insulin or sulfonylurea derivatives (group B) were observed (18). The incidence of influenza was significantly lower in group A $(6 / 110,5.4 \%)$ than in group B $(19 / 79,24 \%)$. This difference was statistically significant ( $\mathrm{P}=0.0003$, Fisher's exact test) (18).

A smaller number of complications following influenza in group A $(1 / 110,0.9 \%)$ as compared with group B (4/79, $5 \%$ ) was not statistically significant $(\mathrm{P}=0.16$, Fisher's exact test) (18).

Biguanides act against other viruses, apart from influenza. For example, polyhexamethylene biguanide exposure has been shown to lead to the viral aggregation of MS2 bacteriophage (19). Persistent interactions between biguanide-based compound NB325 and CXCR4 result in prolonged inhibition of human immunodeficiency virus type 1 infection (20).

\section{Buformin and influenza in mice}

Denys and Bocian examined the protective effect of buformin (Silubin retard) against influenza in mice (21). They used the APR-8 influenza virus strain. The source of the virus was the allantoic fluid of infected chick embryos. They determined, with the method of surviving allantoic sections, that the minimal infectious dilution of influenza virus causing hemagglutination was $10^{-5}$ (Table I). Allantoic sections contain an inhibitor of hemagglutination, and this method is a standard assay of viral infectivity (22).

The buformin preparation was dissolved in phosphate-buffered $0.9 \% \mathrm{NaCl}$ solution. A total of 110 white $\mathrm{BALB} / \mathrm{C}$ mice were used for the experiments, weighing 18-20 g. Mice were infected with $0.05 \mathrm{ml}$ influenza virus intranasally following mild anesthesia. An LD50 infectious dilution assay for mice was carried out and estimated to be $10^{-4}$ (Fig. 1). Half the animals treated died within 10 days after being infected. This titer of APR-8 was used in the buformin studies (21).

The buformin preparation was injected once daily subcutaneously, at a dose of $20 \mathrm{mg} / \mathrm{kg}$, beginning $24 \mathrm{~h}$ after 40 animals had been infected. Treatment was carried out over a period of 4 days. Influenza-infected animals in the control group (40 animals) received $0.9 \% \mathrm{NaCl}$. The observations were carried out over a period of 10 days (Fig. 2). Buformin significantly improved survival $(\mathrm{P}<0.001)$. The buformin=treated mice remained in a much better general condition, compared to the control group that received no buformin. The lungs of buformin-treated mice had less macroscopic inflammation. Further studies indicated that phenformin also improved survival, though to a lesser extent than buformin (23). The buformin results are comparable to the effect of baloxavir marboxil combined with a neuraminidase inhibitor (24).

\section{Inhaled biguanides for influenza}

Inhaling a biguanide for influenza would limit the risk of systemic side-effects associated with biguanides due to the low inhaled dose. Lactic acidosis is the main biguanide systemic side-effect (25). Inhalation would deliver a more 
Table I. Five dilutions of influenza virus tested on 8 allantoic sections.

\begin{tabular}{llllllll}
\hline $10^{-2}$ & Yes & Yes & Yes & Yes & Yes & Yes & Yes \\
$10^{-3}$ & Yes & Yes & Yes & Yes & Yes & Yes & Yes \\
$10^{-4}$ & Yes & Yes & Yes & Yes & Yes & Yes & Yes \\
$10^{-5}$ & Yes & Yes & Yes & Yes & Yes & Yes & Yes \\
$10^{-6}$ & No & No & No & No & No & No & Yes \\
\hline
\end{tabular}

Five dilutions of influenza virus were tested on 8 allantoic sections (40 tests in total). The maximal dilution of influenza virus causing hemagglutination (+) was $10^{-5}$. Allantoic sections contain an inhibitor of hemagglutination, and this method is a standard assay of virus infectivity. The data shown are from the study by Denys and Bocian (21).

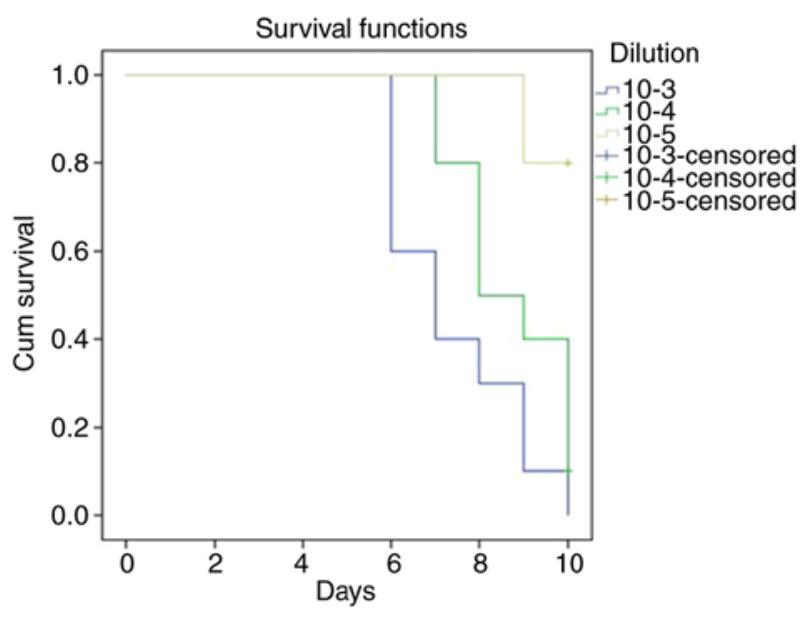

Figure 1. Survival of mice following influenza virus infection with three viral dilutions, 10 mice per dilution group. The effect of dilution was significant $(\mathrm{P}<0.001$, log-rank test). The data shown are from the study by Denys and Bocian (21).

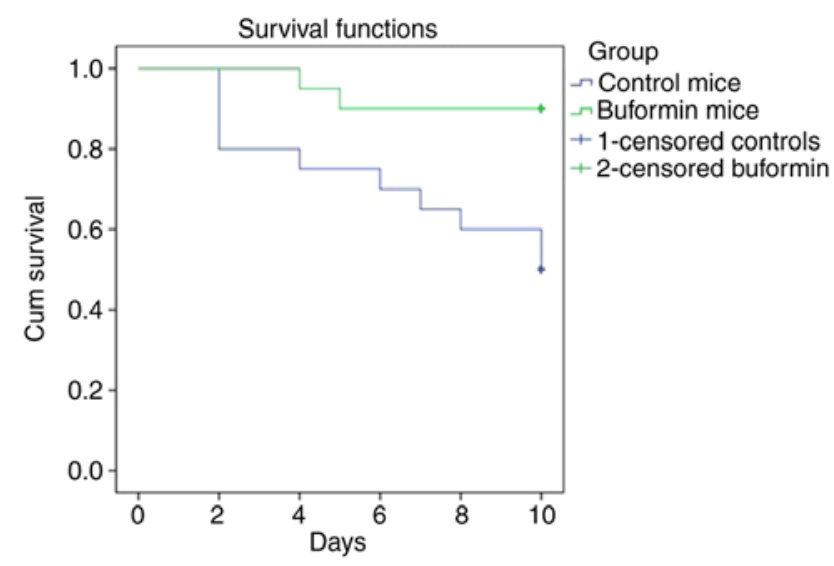

Figure 2. Survival of influenza-infected mice. A total of 40 mice were treated with buformin and 40 mice were used as the untreated controls. The effect of buformin was significant $(\mathrm{P}<0.001, \log$ rank test). The mean survival time for the treated mice was 9.4 days (95\% CI 8.9-9.9). Mean survival of the untreated controls was 7.6 days (95\% CI 6.6-8.7). The data shown are from the study by Denys and Bocian (21).

predictable amount of biguanide to the lung than oral dosing and is an established mode of delivery for a range of therapeutic agents.

Precedence exists for inhaled drug use in influenza. The neuraminidase inhibitor, zanamivir, is administered by

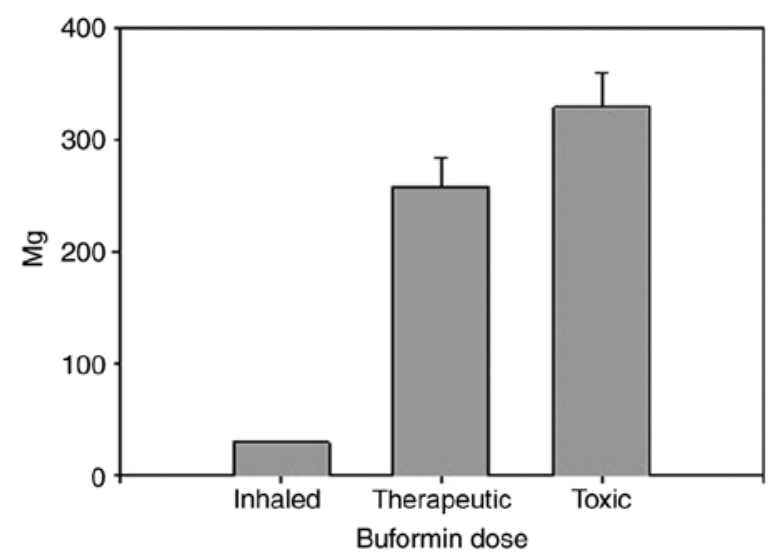

Figure 3. Buformin dosage. The inhaled buformin dose can be increased 10 -fold above what would be needed to treat influenza and would still be well below the systemic toxic dose. This is a key strength of buformin.

inhalation. Inhaled zanamivir requires $10 \mathrm{mg}$ twice a day. The dose of typical inhaled asthma medications is 10-100 $\mu \mathrm{g}$ day.

Oral metformin, then known as flumamine, was examined as an anti-influenza and anti-malarial drug in the Philippines during the late 1940s. Another biguanide anti-malarial drug, biguanil, is still in use (26). A tendency for metformin to lower blood glucose levels in some of the influenza patients was duly noted $(27,28)$.

Metformin is taken orally twice daily by diabetic patients, with a maximum total dose of $2.5 \mathrm{~g} /$ day. Reducing the inhaled dose of an oral drug by a factor of 10-20 typically results in the same local concentration in the airways as by oral administration. Thus, just to equal what the oral dose of metformin would deliver to the airways, a subject would need to inhale 125-250 mg metformin per day; or, if broken into 3 doses/day, 40-80 mg/dose. Delivering this amount of metformin powder to the lungs is at the upper limit of acceptability, and would result in reduced compliance, bronchospasm and cough (29).

Buformin has eight times the potency of metformin. The inhalation of buformin as opposed to metformin, could reduce the dose by a factor of eight. The usual maximum oral dose of buformin is $300 \mathrm{mg} / \mathrm{day}$. Decreasing the inhaled dose by a factor of 10 to 20,3 doses per day inhaled buformin could be administered at 5 to $10 \mathrm{mg}$ per dose, much less than metformin. This dose of buformin, 15 to $30 \mathrm{mg}$ per day, would be highly unlikely to produce lactic acidosis, the main biguanide complication. In a previous study, the toxic oral buformin dose was $329 \pm 30 \mathrm{mg} /$ day in 24 patients who developed lactic 
acidosis while using buformin. Another group of 24 patients administered $258 \pm 25 \mathrm{mg} /$ day buformin did not develop lactic acidosis (25). In other words, the inhaled buformin dose can be increased 10 -fold above what would be needed to treat influenza and would still be well below the systemic toxic dose (Fig. 3). This is a key strength of buformin.

Inhaled buformin has a relatively long lung residence time. Buformin has an octanol/water partition coefficient $(\log \mathrm{P})$ of -1.2 and is hydrophilic. Hydrophilic small molecules with a log P-value $<0$ have a mean lung half-life $\left(\mathrm{t}^{1} / 2\right)$ of approximately $1 \mathrm{~h}$ (29). Hydrophobic small molecules have a mean lung half-life of approximately $1 \mathrm{~min}$ and are less suitable as an influenza treatment because they pass through the lung so rapidly.

A final advantage of buformin over phenformin is that it improves survival of influenza-infected mice with higher efficiency than phenformin (23).

\section{Inhaled buformin or phenformin for coronavirus}

Coronavirus disease 2019 (COVID-19) is an infectious disease caused by SARS-CoV-2, a virus closely related to the SARS virus. The disease is the cause of the 2019-2020 coronavirus outbreak. It is primarily spread between individuals by small droplets emitted from infected individuals when they breathe or cough. The PI3K/AKT/mTOR signaling responses play important roles in MERS-CoV infection and may represent a novel drug target for therapeutic intervention strategies (30). The inhalation of buformin or phenformin for coronavirus may be an effective novel treatment that would limit the risk of systemic side-effects associated with biguanides due to a low inhaled dose.

\section{Conclusions and future perspectives}

The repurposing of old drugs as antivirals holds considerable promise. Statins are a prime example. A randomized placebo-controlled phase II clinical trial (NCT02056340) aimed at evaluating the potential effect of atorvastatin to reduce the severity of illness in influenza-infected patients is currently underway (31). The inhalation of buformin may represent a novel route of administration for an old drug that may also be used a novel treatment strategy for influenza, coronavirus and other viral infections.

\section{Acknowledgements}

Not applicable.

\section{Funding}

No funding was received.

\section{Availability of data and materials}

Not applicable.

\section{Authors' contributions}

SL as the sole author of the present review article was responsible for the conception and design of this article, as well as for the literature search, writing and manuscript preparation and revisions. The author has read and approved the final manuscript.

\section{Ethics approval and consent to participate}

Not applicable.

\section{Patient consent for publication}

Not applicable.

\section{Competing interests}

The authors declare that they have no competing interests.

\section{References}

1. Doyle JD, Chung JR, Kim SS, Gaglani M, Raiyani C, Zimmerman RK, Nowalk MP, Jackson ML, Jackson LA, Monto AS, et al: Interim estimates of 2018-19 seasonal influenza vaccine effectiveness-United States, February 2019. MMWR Morb Mortal Wkly Rep 68: 135-139, 2019.

2. Cohen J: Waning immunity. Science 364: 224-227, 2019.

3. Moscona A: Neuraminidase inhibitors for influenza. N Engl J Med 353: 1363-1373, 2005.

4. O'Hanlon R and Shaw ML: Baloxavir marboxil: The new influenza drug on the market. Curr Opin Virol 35: 14-18, 2019.

5. Takashita E, Ichikawa M, Morita H, Ogawa R, Fujisaki S, Shirakura M, Miura H, Nakamura K, Kishida N, Kuwahara T, et al: Human-to-human transmission of influenza $\mathrm{A}(\mathrm{H} 3 \mathrm{~N} 2)$ virus with reduced susceptibility to baloxavir, Japan, February 2019. Emerg Infect Dis 25: 2108-2111, 2019.

6. Ma XM and Blenis J: Molecular mechanisms of mTOR-mediated translational control. Nat Rev Mol Cell Biol 10: 307-318, 2009.

7. Keating R, Hertz T, Wehenkel M, Harris TL, Edwards BA, McClaren JL, Brown SA, Surman S, Wilson ZS, Bradley P, et al: The kinase mTOR modulates the antibody response to provide cross-protective immunity to lethal infection with influenza virus. Nat Immunol 14: 1266-1276, 2013.

8. Nikolaidis NM, Noel JG, Pitstick LB, Gardner JC, Uehara Y, Wu H, Saito A, Lewnard KE, Liu H, White MR, et al: Mitogenic stimulation accelerates influenza-induced mortality by increasing susceptibility of alveolar type II cells to infection. Proc Natl Acad Sci USA 114: E6613-E6622, 2017.

9. Wang CH, Chung FT, Lin SM, Huang SY, Chou CL, Lee KY, Lin TY and Kuo HP: Adjuvant treatment with a mammalian target of rapamycin inhibitor, sirolimus, and steroids improves outcomes in patients with severe H1N1 pneumonia and acute respiratory failure. Crit Care Med 42: 313-321, 2014.

10. Chuang YC, Ruan SY and Huang CT: Compelling results of adjuvant therapy with sirolimus for severe H1N1 pneumonia. Crit Care Med 42: e687-e688, 2014.

11. Ison MG: Adjuvant immunosuppression in the management of severe influenza: Friend or foe? Crit Care Med 42: 457-459, 2014.

12. Gupta A, Pant G, Mitra K, Madan J, Chourasia MK and Misra A: Inhalable particles containing rapamycin for induction of autophagy in macrophages infected with Mycobacterium tuberculosis. Mol Pharm 11: 1201-1207, 2014.

13. Carvalho SR, Watts AB, Peters JI, Liu S, Hengsawas S, Escotet-Espinoza MS and Williams RO III: Characterization and pharmacokinetic analysis of crystalline versus amorphous rapamycin dry powder via pulmonary administration in rats. Eur J Pharm Biopharm 88: 136-147, 2014.

14. Weiner SM, Sellin L, Vonend O, Schenker P, Buchner NJ, Flecken M, Viebahn R and Rump LC: Pneumonitis associated with sirolimus: Clinical characteristics, risk factors and outcome-a single-centre experience and review of the literature. Nephrol Dial Transplant 22: 3631-3637, 2007.

15. Clements A, Gao B, Yeap SH, Wong MK, Ali SS and Gurney H: Metformin in prostate cancer: Two for the price of one. Ann Oncol 22: 2556-2560, 2011. 
16. Pollak M: Potential applications for biguanides in oncology. J Clin Invest 123: 3693-3700, 2013.

17. Lehrer S, Rheinstein P and Mulshine J: Inhaled buformin for lymphangioleiomyomatosis and early (airway confined) lung cancer. American Association for Cancer Research Annual Meeting Abstracts: 14-A-638-AACR, 2014.

18. Babinski S and Giermaziak H: Influenza epidemic in 1971 in diabetics treated with 1-butyl-biguanidine hydrochloride (Silubin retard) and 1-phenylethyl-biguanidine hydrochloride (Phenformin). Pol Tyg Lek 28: 1815-1817, 1973 (In Polish).

19. Pinto F, Maillard JY, Denyer SP and McGeechan P: Polyhexamethylene biguanide exposure leads to viral aggregation. J Appl Microbiol 108: 1880-1888, 2010.

20. Thakkar N, Pirrone V, Passic S, Keogan S, Zhu W, Kholodovych V, Welsh W, Rando R, Labib M, Wigdahl B and Krebs FC: Persistent interactions between biguanide-based compound NB325 and CXCR4 result in prolonged inhibition of human immunodeficiency virus type 1 infection. Antimicrob Agents Chemother 54: 1965-1972, 2010.

21. Denys A and Bocian J: Effect of Silubin-retard (1-butyl-biguanide hydrochloride) on the course of influenza-virus infection in mice. Pol Tyg Lek 25: 332-334, 1970 (In Polish).

22. Orthel FW: Influenza virus titrations and the inhibitor of hemagglutination in normal allantoic fluid. Arch Gesamte Virusforsch 38: 347-356, 1972.

23. Bocian J, Denys A and Czernek Z: Effect of biguanidine derivatives phenformin and buformin on course of infection with influenza virus in mouse. Dissertationes Pharmaceuticae et Pharmacologicae 23: 581, 1971.

24. Fukao K, Noshi T, Yamamoto A, Kitano M, Ando Y, Noda T, Baba K, Matsumoto K, Higuchi N, Ikeda M, et al: Combination treatment with the cap-dependent endonuclease inhibitor baloxavir marboxil and a neuraminidase inhibitor in a mouse model of influenza A virus infection. J Antimicrob Chemother 74: 654-662, 2019.
25. Luft D, Schmülling RM and Eggstein M: Lactic acidosis in biguanide-treated diabetics: A review of 330 cases. Diabetologia 14 75-87, 1978

26. Garcia Rubino ME, Carrillo E, Ruiz Alcala G, DominguezMartin A, A Marchal J and Boulaiz H: Phenformin as an anticancer agent: Challenges and prospects. Int J Mol Sci 20: E3316, 2019.

27. Garcia EY: Flumamine, a new synthetic analgesic and anti-flu drug. J Philipp Med Assoc 26: 287-293, 1950.

28. Bailey CJ: Metformin: Historical overview. Diabetologia 60: 1566-1576, 2017

29. Patton JS, Fishburn CS and Weers JG: The lungs as a portal of entry for systemic drug delivery. Proc Am Thorac Soc 1: 338-344, 2004

30. Kindrachuk J, Ork B, Hart BJ, Mazur S, Holbrook MR, Frieman MB, Traynor D, Johnson RF, Dyall J, Kuhn JH, et al: Antiviral potential of ERK/MAPK and PI3K/AKT/mTOR signaling modulation for Middle East respiratory syndrome coronavirus infection as identified by temporal kinome analysis. Antimicrob Agents Chemother 59: 1088-1099, 2015.

31. Pizzorno A, Padey B, Terrier O and Rosa-Calatrava M: Drug repurposing approaches for the treatment of influenza viral infection: Reviving old drugs to fight against a long-lived enemy. Front Immunol 10: 531, 2019.

This work is licensed under a Creative Commons Attribution-NonCommercial-NoDerivatives 4.0 International (CC BY-NC-ND 4.0) License. 